\title{
Using Learning Algorithm Behavior to Chart Task Space: The DICES Distance
}

\author{
Adam H. Peterson, \\ adam@axon.cs. byu.edu, \\ Computer Science Department, \\ Brigham Young University, \\ Provo, Utah 84604 \\ Tony R. Martinez, \\ martinez@cs . byu .edu, \\ Computer Science Department, \\ Brigham Young University, \\ Provo, Utah 84604
}

19 Jun 2008

\begin{abstract}
In theory, learning is not possible over all tasks in general. In practice, the tasks for which learning is desired exhibit significant regularity, which makes learning practical. For the most effective learning, it is valuable to understand the nature of this regularity and how it manifests in the tasks where learning is applied. This research presents the DICES distance metric for finding similarity between learning tasks. With this
\end{abstract}


distance metric, a collection of learning tasks can be given a distance matrix. This distance matrix can be used for visualizing the relationships between learning tasks and searching through task space for tasks which are similar in nature. Examples of task visualization are given, and other possible applications of this tool are touched upon.

Keywords: Machine Learning; Meta Learning; Task Similarity; Task Space

\section{Introduction}

Wolpert's no free lunch theorem indicates that in a general sense learning is not possible [11]. Learning algorithms which generalize in a way that improves performance for one class of functions will, at the same time, impair performance for another class. Nevertheless, machine learning has found consistent and significant use in research and industry. The premise of the no free lunch theorem which treats functions as equally likely does not hold in practical application. The class of tasks to which machine learning is routinely applied is much narrower than the set of all functions. Learnable functions correspond at least indirectly to the operations of mechanisms in the real world, and exhibit a large degree of regularity and structure. It is therefore valuable for machine learning approaches to be designed with an understanding of the nature of this collection of learnable functions.

This research presents a method to measure differences in learning tasks as a tool for understanding the relationships between tasks. This tool is used to find a distance matrix between learning tasks drawn from the Irvine Machine Learning Repository. Visualization of the spatial relationships between these tasks is also shown, and the tasks are grouped in clusters. Observations about these tasks are then made using these tools for examining their relationships with each other. 
Although the primary purpose of this research is to examine and understand the nature of learnable tasks and their relationships, this distance metric can be used for other practical purposes. When machine learning research is presented, especially on a new learning algorithm or a variation of an existing one, it is common to validate the research with tables of empirical results. Often the research makes either an overt or implied claim that the proposed algorithm performs well over a diverse collection of learning tasks, and validates it by giving empirical results showing the algorithm performing favorably on the bulk of learning tasks. If care is not taken when selecting learning tasks to ensure that one type of learning task is not over-represented, the results will not actually substantiate this implied claim. Even if the researcher puts forth an effort to use a diverse collection of learning tasks, it is not always easy to determine whether two learning tasks are similar in nature and thus potentially redundant. With a better understanding of how learning tasks are distributed and related, researchers can select tasks with the objective of thoroughly covering the field of learnable tasks.

At other times, a researcher's goal may be the opposite. Having constructed a new algorithm or method, she carefully determines or hypothesizes what types of learning tasks will benefit from it, and wishes to gather a collection of learning tasks of this nature. In this case, the same difficulty occurs in reverse - it is not always evident whether two learning tasks are similar. It is desirable to be able to find learning tasks which are related, and the research presented here can provide tools for doing this.

\section{Related Work}

Prior work in the area of relating learning tasks includes work by Pfahringer et al on Landmarking [7]. Landmarking positions tasks in a task space by executing 
simple learning models on them and measuring the performance of the models. The research presented herein is inspired by Landmarking, although tasks are related by the behavior of learning models, rather than the performance of models as in Landmarking. In the Landmarking system, rules are induced (e.g. if algorithm $A$ does better than algorithm $B$, then algorithm $C$ will do better than algorithm $D$ ) and the objective is to determine which learning algorithms to employ based on the performance of these markers. The focus of the research presented here is on finding relationships and understanding the structure of "task-space" and how tasks relate to each other.

The task of matching learning algorithms to problems is approached by Brodley [3]. Brodley's work uses ambitious heuristics to try to match a learning model to a task. As with Landmarking, Brodley's work is focused on producing rules and heuristics to match a learning task to an appropriate algorithm to maximize accuracy. Brodley measures characteristics of the learning task rather than using simple algorithms as markers.

One of the ultimate goals of this research is to provide a more robust and sound way of supporting research through empirical results. Aha [1] scrutinizes empirical case studies with a similar goal. Aha's emphasis is to encourage the use of a more rigorous framework for case studies to improve the quality of the results being presented.

The concept of task similarity is valuable in the context of transfer learning and lifelong learning, such as is given by Thrun [10, 9]. Thrun demonstrates that learning incrementally across multiple task domains is effective. Thrun also presents a hierarchical clustering approach for learning tasks called $T C$, or Task Clustering [8]. Thrun's TC approach is focused on transfer learning and clusters learning tasks based on the potential for a model trained on one task to be able to effectively represent another. 
Baxter [2] discusses lifelong learning with an emphasis on using related learning tasks. Baxter posits that lifelong learning is most effective when the same internal representation can be used for several related tasks. The distance metric presented in this research can be used as a tool to discover and quantify the relatedness of tasks based on their learnable regularity.

\section{Measuring Learning Task Similarity}

The tasks toward which machine learning is applied all have some degree of regularity and structure to them. It is this structure which makes learning possible. Learning algorithms exploit the structure to perform predictions and classifications. Many learning algorithms have been developed in machine learning over several decades. Some algorithms recognize one type of structure, while others recognize another. It is an open question whether all types of regularity occurring in learnable tasks have been exploited by some algorithm, and it will probably remain an open question for the foreseeable future. Regardless, a sizable collection of diverse learning algorithms with different strengths and weaknesses is available today.

Different professionals approach machine learning with different goals. Some have a specific set of learning tasks that are important to them and are always looking for ways to improve performance on these particular tasks. Others are researchers, working to create and improve learning algorithms to cover a wide variety of learning tasks and achieve improved performance over them generally. Yet other researchers are interested in creating and reasoning about cognitive models that exhibit general problem solving and adaptability inspired by human intelligence. As different people have different goals, it can be difficult for one practitioner to know when the published work of another is relevant to her own objectives. A method to determine how related two learning tasks are can be a 
valuable tool for a professional to evaluate the usefulness of published results.

More generally, it is valuable to understand the relationship between learning tasks in order to better understand what makes them learnable. With better understanding of how learnable tasks relate to each other and how they differ from the general class of functions Wolpert discusses as unlearnable, the task of learning in general can be approached more effectively.

This research provides such a tool for determining the relationships between learning tasks.

\subsection{The COD Metric For Algorithm Behavior Variation}

The distance metric for learning tasks presented in this research uses the COD (Classifier Output Difference) distance measure for learning algorithms by Peterson [6] as a tool. The problem of learning task similarity is approached here by examining the effect learning tasks have on the behavior of the models produced by learning algorithms. Given a learning task and two learners, the COD metric is a measure of the extent to which the learners produce differing predictions. More specifically, it is an estimate of the probability that, given a new instance, the learners will classify the instance differently. Algorithm 1 describes how the COD distance is estimated. In essence, the two algorithms are used to produce hypotheses from a classification training set, and the frequency of disagreement of those algorithms on the test set is measured.

\subsection{From Algorithm Diversity to Learning Task Diversity}

The core contribution of the research given here is a way to characterize and measure the similarity and difference between learning tasks from a learnability standpoint. Tasks are considered similar if they elicit similar relative behavior characteristics from learning algorithms. The COD distance is used to quan- 


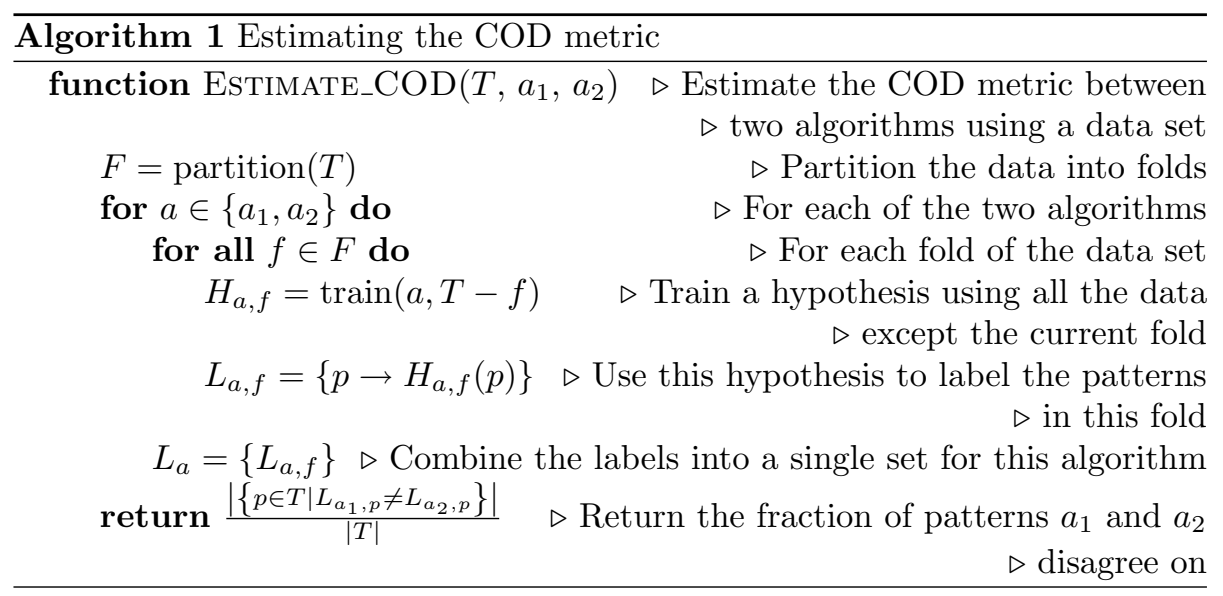

tify the behavior characteristics of a task with respect to a learning algorithm. When two tasks have similar observed effects on the models produced by various algorithms, this provides evidence that the tasks have similar types of learnability.

For example, if task $t_{1}$ results in a small COD distance between algorithms $a_{1}$ and $a_{2}$, but a large distance between algorithms $a_{2}$ and $a_{3}$, while task $t_{2}$ similarly gives a small COD distance between $a_{1}$ and $a_{2}$ and a large distance between $a_{2}$ and $a_{3}$, this indicates that the tasks bear similarity to each other in terms of their "learnable potential." (Recall that when using the COD metric, algorithm distances are measures of behavior difference.) If, on the other hand, $t_{3}$ gives a large distance between $a_{1}$ and $a_{2}$, but a small distance between $a_{2}$ and $a_{3}$, this indicates that there is something about learning task $t_{3}$ that is different in character from $t_{1}$ and $t_{2}$. In this research, relative COD distances are compared rather than absolute distances because the COD distance will have a tendency to be smaller for tasks that are simpler (have less process noise, fewer outliers, etc.) simply because there are fewer missed patterns for algorithms to disagree on, and using relative distances can reduce this effect.

One may observe that the term "learnable potential" used here does not 


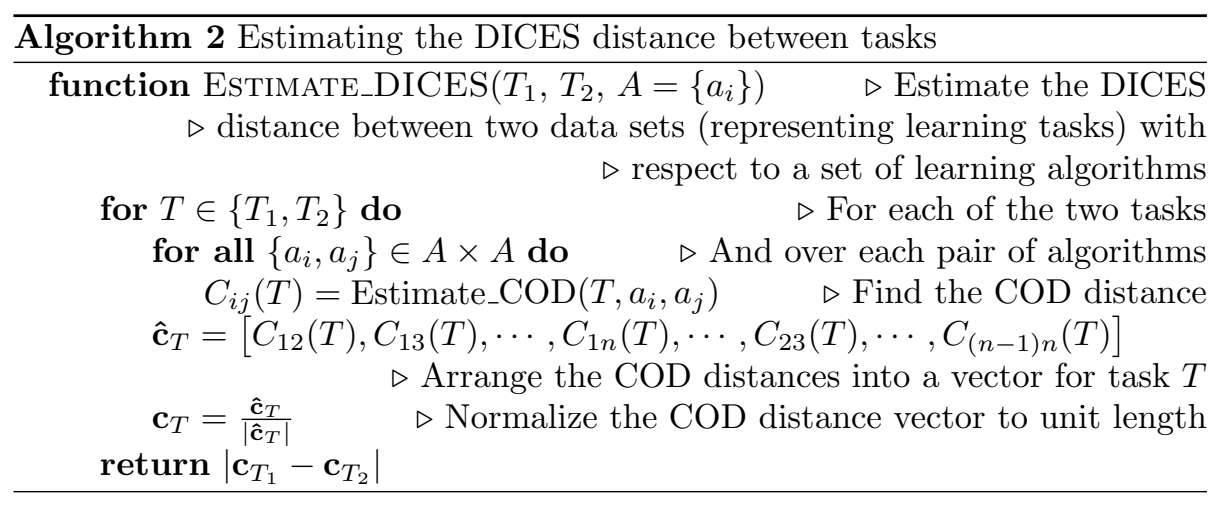

have a strict definition. Conceptually, this term relates to the aspects of a task that make it learnable, the qualities of regularity contained within it. This term can not be defined formally; otherwise, the definition could be used to characterize the circumstances under which learning is possible, which does not unify well with Wolpert's No Free Lunch theorem. However, although the learnable character of a learning task may be difficult to formulate, it is still valuable to make an effort to understand some of its properties and aspects. By observing which tasks produce similar effects on COD algorithm distances and which produce differing effects, one can begin to quantify the similarity of learning tasks. This quantification can lead to observing learning tasks in quasi-spatial relationships.

We now present the Difference In COD Estimate Similarities (DICES) distance measurement between learning tasks. The procedure for computing the DICES metric between two tasks is given in Algorithm 2, and outlined here:

1. A set of COD distances between algorithms on each task is computed. The COD distance between algorithms $a_{1}$ and $a_{2}$, members of a set of algorithms $A$, over a task $t$ is denoted as $C_{a_{1}, a_{2}}(t)$.

2. These pairwise COD distances are then placed a vector of distances for 
each task:

$$
\begin{aligned}
\hat{\mathbf{c}}_{A}(t)=[ & C_{a_{1}, a_{2}}(t), C_{a_{1}, a_{3}}(t), \cdots, C_{a_{1}, a_{n}}(t), \\
& C_{a_{2}, a_{3}}(t), \cdots, C_{a_{2}, a_{n}}(t), \\
& \left.\cdots, C_{a_{n-1}(t), a_{n}}\right]
\end{aligned}
$$

where $n=|A|$.

3. The vector of distances is then normalized:

$$
\mathbf{c}_{A}(t)=\frac{\hat{\mathbf{c}}_{A}(t)}{\left|\hat{\mathbf{c}}_{A}(t)\right|}
$$

4. Finally, the distance between the two tasks $t_{1}$ and $t_{2}$ is calculated by measuring the length of the difference between their normalized COD vectors:

$$
D_{A}\left(t_{1}, t_{2}\right)=\left|\mathbf{c}_{A}\left(t_{1}\right)-\mathbf{c}_{A}\left(t_{2}\right)\right|
$$

The intuitive basis for measuring learning task similarity in this manner is related to the idea that learnable problems exhibit regularity and structure that is exploited by learning algorithms to improve predictive accuracy. It is this structure in a learning task which makes a task learnable. Without some amount of regularity and structure in a task, the premise of the No Free Lunch Theorem applies. No assumptions can be made about the task, and the task can not be effectively learned. Learnable tasks, on the other hand, have significant structure that can be detected by learning algorithms and used to perform generalization.

Different kinds of structure are found in different learning tasks, and different learning algorithms exploit this regularity in different ways. For example, if one is given the learning task of predicting how crowded a swimming pool 
will be from the outside temperature that day, a linear learning model such as a perceptron will attempt to determine a correlation between temperature and patronage, and output a model that predicts large crowds on one side of a threshold and small crowds on the other. An instance based model, on the other hand, will use the premise that past examples will likely be similar to future instances with similar temperatures, and will make a prediction based on previous days with similar temperatures. Although both learning algorithm types are able to produce a model for predicting the target, these models function in different ways, exploiting the regularity of the target problem differently.

In some cases, the different ways two algorithms exploit task regularity will lead to models with very different behaviors. In other cases, the difference in model representation will be less relevant and still result in learning models that have similar behaviors. Because different models represent different kinds of regularity and in different ways, these behavioral differences between models can serve as an indirect indicator of what types of regularity a learning task contains. Intuitively, if two learning tasks have regularity of a similar nature to one another, different learning algorithms will capture this regularity in each task in a similar way. We can infer similarity between two tasks from similar patterns in the COD measurements they induce in learning algorithms.

The DICES distance between a task and itself will always be zero, since each learning algorithm (as executed on deterministic hardware) will induce the same hypothesis when given the same data set. Some learning algorithms, such as multilayer perceptrons, Boltzmann machines, and random forests, employ pseudo-random sources to set initial conditions or govern other aspects of the training process. Algorithms with high variance can exhibit a high sensitivity to resampling or presentation order. By varying random sources, sampling, and presentation order, algorithms may induce hypotheses with nonzero COD 
distances to each other. The characteristics of these distances and the resulting DICES distances are potentially interesting, but beyond the scope of this work.

In this research, a learning task is characterized by a data set drawn from the task. There are domains of machine learning in which the data set is not constant, such as Active Learning [4] where the learning algorithm has the option of querying an expert on unseen patterns. Such domains are interesting, but beyond the scope of this research. Applying the DICES distance in such domains may be a future research direction, if the COD distance (or an analog) can be formulated between Active Learning tasks or other tasks not directly characterizable by a data sampling.

\section{Results And Analysis}

With the DICES measure, a distance matrix can be constructed for a collection of tasks, and visualization and clustering of the task space can be performed. Table 1 lists 30 tasks from the Irvine Machine Learning Repository [5] over which DICES distances were measured. The 18 learning algorithms used for these DICES measures are listed in Table 2.

The computed DICES distances between the tasks are given in Table 3. The average distance between two tasks is 0.058 and the table has bolded entries for any distance less than 0.030 , one standard deviation less than the average. One quirk of this collection of data sets, which is an example of why examining redundancy in a collection of data sets can be useful, is that four pairs of data sets are each essentially the same task, with either a change in the way the inputs or outputs are encoded, or the sampling order. The stgern and stgers tasks are the same except that in the second some of the inputs have been re-encoded

as continuous rather than nominal. The cmuvow and vowel tasks are the same task except that the output has been encoded as a 1-to-10 discrete value in one 
and a 1-of-10 nominal value in the other, and in one data set about $40 \%$ of the patterns have been removed. The tasks stsegm and segm are the same task, except that in one data set, the output has been coded as a nominal value with seven classes, and in the other the patterns are marked with a discrete value between 1 to 7 . The tasks cmuson and sonar are both the rocks-and-mines problem, with the only difference being that in one the rock is class 1 and in the other the mine is class 1.

These observations are all reflected in the DICES distance matrix. Four of the five shortest distances are the four task pairs mentioned. The shortest distance is 0.002 , between stsegm and segm. The distance between cmuson and sonar is 0.005 , the second shortest distance in the table. The distance between cmuvow and vowel is 0.013, which is the third shortest distance of the table. The fifth shortest distance in the matrix is 0.017 between stgern and stgers. All four of these distances are well below the 0.030 value of the mean minus a standard deviation.

Another observation that can be drawn is that the data sets tend to form cliques. For example, the glass data set is near the bupa, cmuson, cmuvow, sonar, and shvehi data sets (with distances less than 0.25). Moving over to the bupa column, it can be seen that this data set is also close to cmuson, glass, sonar, staust, and stvehi. Three of the four tasks closest to glass (not counting bupa) are also three of the four closest tasks to bupa (not counting glass).

Another clique can be observed by looking at the near neighbors of pima, which are: cmuson, sonar, stgern, stgers, and wave21. The neighbors of wave21 are: cmuson, pima, sonar, stgern, and stgers. In this case, all four of the nearest neighbors are common between these two tasks, pima, and wave21. 


\subsection{Visualizing Task Relatonships}

It is insightful to observe a visual representation of relationships between entities. Although the DICES metric is a Euclidean distance measure, this Euclidean space is of very high dimension. Because there are 18 algorithms, there are $\frac{17 \cdot 18}{2}=154$ components to the vector, which means that the distance is actually in a 154 dimensional space. However, as there are only 30 tasks to visualize, a 29-dimensional subspace will actually suffice to represent all distances with full fidelity. Unfortunately, 29 dimensions is still much higher than we have convenient visualization tools. Still, an approximate visualization can be shown in three dimensional space, by placing points representing tasks into 3-space and then updating their positions with gradient-descent to match the distance matrix.

Such a visualization is shown in Fig. 1. In this figure, the nodes have been given colorations to help distinguish the structure of the representation. The coloration is chosen based on average distance to the rest of the tasks, with darker nodes having shorter average distances to all other tasks and lighter nodes having longer average distances.

The visualization shows, as one would expect, the four "pair" (stgern and stgers, cmuson and sonar, stsegm and segm, and cmuvow and vowel) tasks close together. In fact, stsegm and segm are virtually on top of each other, as are cmuson and sonar. On the other hand, there are several tasks which are strongly distinctive, which tend to hover on the outskirts of the space. The overall layout of this space appears to be a dense cluster of tasks in the middle of the space and a number of other tasks loosely grouped around it.

By viewing this learning task structure in three dimensions instead of 29, we do lose some information. It is useful to consider how much information is being lost. For some insight into this issue, one can look at the eigensystem of 
the covariance matrix of the higher dimensional space. The sorted eigenvalues are shown in Fig. 2. Here, one can see that there are two strong eigenvalues, representing two dimensions of high information content, followed by a number of rather small eigenvalues. From this it can be inferred that a two dimensional representation is enough to capture a large amount of the structure. Thus, the three dimensional representation can give a reasonable picture.

\subsection{Task Relationships Through Clustering}

With a distance matrix over learning tasks, it is possible to do various forms of analysis on the task relationships, such as cluster analysis. Fig. 3 shows a dendrogram of learning tasks from doing average link agglomerative clustering. The nodes of this figure are colored to match the colors used in Fig. 1. In this figure, we can observe that the cliques that were noted in the beginning of section 4 have formed clusters. The first clique that was mentioned consisted of glass, bupa, cmuson, sonar, and stvehi. In Fig. 3, we observe that with the addition of stsati these tasks all form a cluster. The second clique observed consisted of pima, cmuson, sonar, stgern, stgers, and wave21. Although cmuson and sonar are part of the first cluster, the other four members of this clique form a cluster in the dendrogram.

We may also observe in this dendrogram that the darker central nodes agglomerate to form a single large cluster, with the lighter exterior nodes forming their own clusters. It appears that in the collection of Irvine MLDB tasks selected, there exists a relatively dense clustering of tasks about a well-defined center. If this result generalizes to learning tasks in general, it suggests that learning algorithms which focus on this part of the task space will tend to improve performance on a large number of learning tasks. Conversely, algorithms focused on performance on the outlying tasks will tend to cover fewer learning 
tasks unless they have a broad coverage of the task space in these outlying areas.

To verify the robustness of this approach, other clustering techniques were also applied to these tasks. These clustering methods included minimum link agglomerative clutering and recursive partitioning using maximum graph cut. The observations found in these clusterings were similar to those made above with average link agglomerative clustering.

\section{Conclusion and Future Work}

This research provides a method for computing the distances between learning tasks. This is done by building on the concept of an algorithm distance and reversing it to allow for distances between tasks. This research gives examples of visualizations for use in understanding the relationships between learning tasks.

Future work in this area includes research into uniqueness functions that can be used to weight data sets for empirical results. With the ability to generate a distance matrix, this work may also be applied to transfer learning.

Some work has been done on using distance relationships between tasks to guide task selection for presenting empirical results across a broad range of tasks while minimizing redundancy in tasks (that is, tasks which are closely related). Work is also being done in producing weightings for empirical results which can be applied to already published research in order to determine the broadness of the algorithm(s) presented by them.

Other future directions for this research include examining task space from the perspective of algorithm coverage and determining where individual learning algorithms perform well or poorly in relation to tasks and to each other. It is hoped that in being able to visualize and understand learning task space better, research might be made more effective at targeting unexplored techniques for machine learning. 


\section{References}

[1] D. W. Aha. Generalizing from case studies: A case study. In Proceedings of the Ninth International Conference on Machine Learning, ICML'2000, pages 1-10, Aberdeen, Scotland, 1992. Morgan Kaufmann.

[2] J. Baxter. Learning model bias. In D. S. Touretzky, M. C. Mozer, and M. E. Hasselmo, editors, Advances in Neural Information Processing Systems, volume 8, pages 169-175. The MIT Press, 1996.

[3] C. E. Brodley. Dynamic automatic model selection. Technical Report UMCS-1992-030, University of Massachusetts, February 1992.

[4] D. Cohn, L. Atlas, and R. Ladner. Improving generalization with active learning. Machine Learning, 15(2):201-221, 1992.

[5] C. J. Merz and P. M. Murphy. UCI repository of machine learning databases. Available at http://www.ics.uci.edu/ mlearn/ MLRepository.html, 1996.

[6] A. H. Peterson and T. R. Martinez. Estimating the potential for combining learning models. In Proceedings of the ICML Workshop on Meta-Learning, pages 68-75, 2005.

[7] B. Pfahringer, H. Bensusan, and C. Giraud-Carrier. Meta-learning by landmarking various learning algorithms. In Proceedings of the Seventeenth International Conference on Machine Learning, ICML'2000, pages 743-750. Morgan Kaufmann, San Francisco, California, 2000.

[8] S. Thrun and J. O'Sullivan. Discovering structure in multiple learning tasks: The TC algorithm. In International Conference on Machine Learning, pages 489-497, 1996. 
[9] S. B. Thrun. Is learning the $n$-th thing any easier than learning the first? In D. S. Touretzky, M. C. Mozer, and M. E. Hasselmo, editors, Advances in Neural Information Processing Systems, volume 8, pages 640-646. The MIT Press, 1996.

[10] S. B. Thrun and T. M. Mitchell. Learning one more thing. In IJCAI, pages 1217-1225, 1995.

[11] D. H. Wolpert and W. G. Macready. No free lunch theorems for search. Technical Report SFI-TR-95-02-010, Santa Fe Institute, Santa Fe, New Mexico, 1995. 
Table 1: Learning tasks used in this research and some of their characteristics.

\begin{tabular}{|lrrrrrrr|}
\hline Data Set & Instances & Classes & Features & Bool. & Enum. & Disc. & Cont. \\
\hline ann & 7200 & 3 & 21 & 15 & & & 6 \\
breastw & 699 & 2 & 9 & & & 9 & \\
bupa & 345 & 2 & 6 & & & & 6 \\
cmuson & 208 & 2 & 60 & & & & 60 \\
cmuvow & 990 & 6 & 10 & & & 10 & \\
dermat & 366 & 6 & 34 & 1 & & 32 & 1 \\
ecoli & 336 & 8 & 7 & & & & 7 \\
glass & 214 & 7 & 9 & & & & 9 \\
iono & 351 & 2 & 34 & & & & 34 \\
iris & 150 & 3 & 4 & & & & 4 \\
led7 & 200 & 10 & 7 & 7 & & & \\
lymph & 148 & 4 & 18 & 9 & & 9 & \\
musk1 & 476 & 2 & 166 & & & & 166 \\
newthy & 215 & 3 & 5 & & & & 5 \\
pima & 768 & 2 & 8 & & & & 8 \\
promot & 106 & 2 & 57 & & 57 & & \\
segm & 2310 & 7 & 19 & & & & 19 \\
sonar & 208 & 2 & 60 & & & & 60 \\
splice & 3190 & 3 & 60 & & 60 & & \\
staust & 690 & 2 & 14 & 4 & & 4 & 6 \\
stgern & 1000 & 2 & 24 & & & & 24 \\
stgers & 1000 & 2 & 20 & & 13 & & 7 \\
sthear & 270 & 2 & 13 & 3 & 1 & 4 & 5 \\
stsati & 6435 & 6 & 36 & & & & 36 \\
stsegm & 2310 & 7 & 19 & & & & 19 \\
stvehi & 846 & 4 & 18 & & & & 18 \\
vowel & 990 & 6 & 10 & & & & 21 \\
wave21 & 300 & 3 & 21 & & & & \\
wine & 178 & 3 & 13 & & & & \\
zoo & 101 & 7 & 16 & 15 & 1 & & \\
\hline & & 310 & & & & \\
\end{tabular}

Columns in order are: Data set name, instance count, output class count, input feature count, boolean inputs, enumerated inputs, discrete inputs, and continuous inputs. 
Table 2: List of algorithms used for the DICES measurements.

\begin{tabular}{|ll|}
\hline Algorithm & Family \\
\hline C4.5 & Decision tree \\
ID3 expect & Decision tree \\
ID3 gain & Decision tree \\
ID3 ratio & Decision tree \\
1-NN & Instance based \\
5-NN & Instance based \\
KStar & Instance based \\
Logistic & Regression \\
JRip & Rule based \\
NB Gaussian & Naïve Bayes \\
NB class seg & Naïve Bayes \\
NB input seg & Naïve Bayes \\
NB Weka & Naïve Bayes \\
MLP & Neural network \\
RBF & Neural network \\
SLP & Neural network \\
SVM & Support vector \\
SVM radial & Support vector \\
\hline
\end{tabular}


Table 3: The distance between each learning task, using the DICES metric. The table is symmetric, so only the lower half is shown. It is split horizontally to fit on the page. The mean distance between two tasks is 0.580 , and the standard deviation is 0.215 .

\begin{tabular}{|c|c|c|c|c|c|c|c|c|c|}
\hline & $(\mathbf{A N})$ & & & & & & & & \\
\hline breastw & (BR) & 0.605 & & & & & & & \\
\hline bupa & $(B U)$ & 0.466 & 0.495 & & & & & & \\
\hline cmuson & (CS) & 0.497 & 0.442 & 0.215 & & & & & \\
\hline cmuvow & $(\mathrm{CV})$ & 0.549 & 0.585 & 0.392 & 0.366 & & & & \\
\hline dermat & (DE) & 0.597 & 0.327 & 0.419 & 0.386 & 0.537 & & & \\
\hline ecoli & (EC) & 0.707 & 0.791 & 0.578 & 0.552 & 0.499 & 0.679 & & \\
\hline glass & (GL) & 0.467 & 0.567 & 0.248 & 0.272 & 0.283 & 0.515 & 0.504 & \\
\hline iono & (IO) & 0.831 & 0.875 & 0.666 & 0.657 & 0.725 & 0.745 & 0.558 & 0.739 \\
\hline iris & (IR) & 0.688 & 0.760 & 0.581 & 0.545 & 0.460 & 0.642 & 0.399 & 0.548 \\
\hline led 7 & $(\mathrm{~L} 7)$ & 0.692 & 0.868 & 0.738 & 0.729 & 0.547 & 0.841 & 0.627 & 0.581 \\
\hline lymph & $(\mathrm{LY})$ & 0.539 & 0.288 & 0.392 & 0.333 & 0.498 & 0.363 & 0.715 & 0.451 \\
\hline musk1 & (M1) & 0.551 & 0.564 & 0.465 & 0.453 & 0.596 & 0.548 & 0.701 & 0.511 \\
\hline new thy & (NE) & 0.658 & 0.813 & 0.655 & 0.648 & 0.470 & 0.779 & 0.541 & 0.502 \\
\hline pima & (PI) & 0.457 & 0.485 & 0.342 & 0.264 & 0.403 & 0.429 & 0.508 & 0.339 \\
\hline promot & (PR) & 0.524 & 0.359 & 0.452 & 0.412 & 0.582 & 0.386 & 0.755 & 0.521 \\
\hline segm & (SE) & 0.965 & 1.005 & 0.789 & 0.785 & 0.835 & 0.869 & 0.607 & 0.847 \\
\hline sonar & (SO) & 0.498 & 0.439 & 0.218 & 0.066 & 0.368 & 0.390 & 0.549 & 0.277 \\
\hline splice & (SP) & 0.528 & 0.567 & 0.596 & 0.599 & 0.762 & 0.643 & 0.875 & 0.663 \\
\hline staust & (SA) & 0.528 & 0.539 & 0.282 & 0.309 & 0.515 & 0.477 & 0.676 & 0.358 \\
\hline stgern & $(\mathrm{SN})$ & 0.467 & 0.424 & 0.325 & 0.256 & 0.457 & 0.391 & 0.596 & 0.369 \\
\hline stgers & (SS) & 0.482 & 0.405 & 0.362 & 0.302 & 0.471 & 0.386 & 0.662 & 0.408 \\
\hline sthear & $(\mathrm{SH})$ & 0.547 & 0.368 & 0.436 & 0.382 & 0.581 & 0.364 & 0.738 & 0.511 \\
\hline stsati & (ST) & 0.578 & 0.535 & 0.294 & 0.249 & 0.411 & 0.408 & 0.507 & 0.390 \\
\hline stsegm & (SG) & 0.965 & 1.006 & 0.790 & 0.787 & 0.836 & 0.871 & 0.608 & 0.848 \\
\hline stvehi & $(\mathrm{SV})$ & 0.516 & 0.532 & 0.282 & 0.262 & 0.320 & 0.456 & 0.497 & 0.239 \\
\hline vowel & (VO) & 0.574 & 0.667 & 0.467 & 0.446 & 0.140 & 0.616 & 0.484 & 0.313 \\
\hline wave 21 & $(\mathrm{WV})$ & 0.462 & 0.351 & 0.326 & 0.267 & 0.450 & 0.319 & 0.601 & 0.402 \\
\hline & (WI) & 0.836 & 1.006 & 0.860 & 0.840 & 0.646 & 0.954 & 0.595 & 0.708 \\
\hline \multirow[t]{2}{*}{ zoo } & $(\mathrm{ZO})$ & 0.694 & 0.759 & 0.663 & 0.638 & 0.448 & 0.697 & 0.532 & 0.522 \\
\hline & & $(\mathbf{A N})$ & (BR) & $(\mathrm{BU})$ & $(\mathrm{CS})$ & $(\mathrm{CV})$ & (DE) & (EC) & (GL) \\
\hline
\end{tabular}

\begin{tabular}{|c|c|c|c|c|c|c|c|c|c|c|c|}
\hline $\begin{array}{l}\text { (IR) } \\
(\mathbf{L} 7)\end{array}$ & $\begin{array}{l}0.506 \\
0.955\end{array}$ & 0.666 & & & & & & & & & \\
\hline $\begin{array}{l}(L 7) \\
(L Y)\end{array}$ & $\begin{array}{l}0.955 \\
0.823\end{array}$ & $\begin{array}{l}0.660 \\
0.686\end{array}$ & 0.794 & & & & & & & & \\
\hline & 0.823 & & & & & & & & & & \\
\hline & $\begin{array}{l}0.774 \\
0.879\end{array}$ & 0.722 & 0.847 & 0.746 & 0.782 & & & & & & \\
\hline (PI) & $\begin{array}{l}0.879 \\
0.707\end{array}$ & 0.588 & 0.260 & 0.376 & 0.452 & 0.571 & & & & & \\
\hline & & 0.504 & $=0.632$ & & & & & & & & \\
\hline (SE) & $\begin{array}{l}0.844 \\
\mathbf{0 . 2 4 0}\end{array}$ & 0.717 & 0.838 & 0.329 & 0.519 & 0.810 & 0.395 & 0 & & & \\
\hline (SO) & $\begin{array}{l}0.240 \\
0.661\end{array}$ & $\begin{array}{l}0.611 \\
0.557\end{array}$ & $\begin{array}{l}1.041 \\
0.741\end{array}$ & $\begin{array}{l}0.953 \\
0.329\end{array}$ & 0.453 & $\begin{array}{l}0.972 \\
0.661\end{array}$ & $\begin{array}{r}0.838 \\
\mathbf{0 . 2 7 3}\end{array}$ & $\begin{array}{ll}3 & 0.970 \\
3 & 0.413\end{array}$ & 0.784 & & \\
\hline$(\mathbf{S P})$ & 0.935 & 0.881 & 0.893 & 0.540 & 0.535 & 0.899 & 0.558 & 0.426 & 1.063 & 0.599 & \\
\hline (SA) & 0.777 & 0.693 & 0.810 & 0.386 & 0.427 & 0.749 & 0.393 & 0.461 & 0.883 & 0.307 & 0.585 \\
\hline (SN) & 0.761 & 0.600 & 0.741 & 0.325 & 0.443 & 0.686 & 0.194 & 40.308 & 30.891 & 0.256 & 0.500 \\
\hline (SS) & 0.797 & 0.635 & 0.721 & 0.282 & 0.423 & 0.676 & 0.234 & 0.317 & 0.932 & 0.312 & 0.507 \\
\hline$(\mathrm{SH})$ & 0.844 & 0.699 & 0.835 & 0.311 & 0.445 & 0.774 & 0.334 & 0.330 & 0.974 & 0.388 & 0.543 \\
\hline (ST) & 0.499 & 0.464 & 0.791 & 0.429 & 0.503 & 0.718 & 0.352 & 0.483 & 0.620 & 0.245 & 0.678 \\
\hline (SG) & 0.240 & 0.611 & 1.043 & 0.954 & 0.901 & 0.973 & 0.839 & 0.971 & 0.014 & 0.785 & 1.064 \\
\hline (SV) & 0.685 & 0.537 & 0.661 & 0.427 & 0.493 & 0.609 & 0.353 & $\begin{array}{ll}3 & 0.490\end{array}$ & 0.787 & 0.258 & 0.652 \\
\hline (VO) & 0.764 & 0.476 & 0.444 & 0.581 & 0.650 & 0.367 & 0.450 & 0.658 & 0.864 & 0.453 & 0.812 \\
\hline (WV) & 0.701 & 0.565 & 0.744 & 0.297 & 0.385 & 0.680 & 0.245 & 50.323 & 0.845 & 0.272 & 0.504 \\
\hline (WI) & 0.929 & 0.642 & 0.297 & 0.944 & 0.942 & 0.319 & $\begin{array}{ll}9 & 0.749\end{array}$ & 0.984 & 0.989 & 0.852 & 1.047 \\
\hline \multirow[t]{2}{*}{$(\mathrm{ZO})$} & 0.869 & 0.571 & 0.323 & 0.704 & 0.786 & 0.320 & 0.588 & 0.765 & 0.960 & 0.649 & 0.891 \\
\hline & (IO) & (IR) & (L7) & (LY) & (M1) & (NE) & (PI) & (PR) & (SE) & $(\mathrm{SO})$ & (SP) \\
\hline$(\mathrm{SN})$ & 0.347 & & & & & & & & & & \\
\hline (SS) & 0.334 & 0.199 & & & & & & & & & \\
\hline$(\mathrm{SH})$ & 0.382 & 0.279 & 0.215 & & & & & & & & \\
\hline$(\mathrm{ST})$ & 0.397 & 0.372 & 0.410 & 0.474 & & & & & & & \\
\hline (SG) & 0.884 & 0.891 & 0.933 & 0.975 & 0.621 & & & & & & \\
\hline (SV) & 0.380 & 0.374 & 0.412 & 0.488 & 0.315 & 0.789 & & & & & \\
\hline (VO) & 0.582 & 0.530 & 0.537 & 0.654 & 0.501 & 0.865 & 0.384 & & & & \\
\hline$(\mathrm{WV})$ & 0.392 & 0.241 & 0.245 & 0.264 & 0.335 & 0.846 & 0.351 & 0.526 & & & \\
\hline (WI) & 0.938 & 0.872 & 0.870 & 0.966 & 0.873 & 0.990 & 0.774 & 0.535 & 0.867 & & \\
\hline \multirow[t]{2}{*}{ (ZO) } & 0.750 & 0.679 & 0.665 & 0.764 & 0.694 & 0.962 & 0.569 & 0.369 & 0.666 & 0.383 & \\
\hline & (SA) & (SN) & (SS) & $(\mathrm{SH})$ & (ST) & (SG) & $(\mathrm{SV})$ & $(\mathrm{VO})$ & $(\mathrm{WV})$ & (WI) & $(\mathrm{ZO})$ \\
\hline
\end{tabular}




\section{List of Figures}

1 A visualization of task space in three dimensions for thirty tasks from the Irvine Machine Learning Repository. Two views of the model are shown, roughly 90 degrees apart. . . . . . . . . . . 22

2 The eigenvalues of the task space's covariance matrix. . . . . . . 23

3 Clustering of learning tasks using Average Link Agglomerative Clustering. . . . . . . . . . . . . . . . . . . 24 

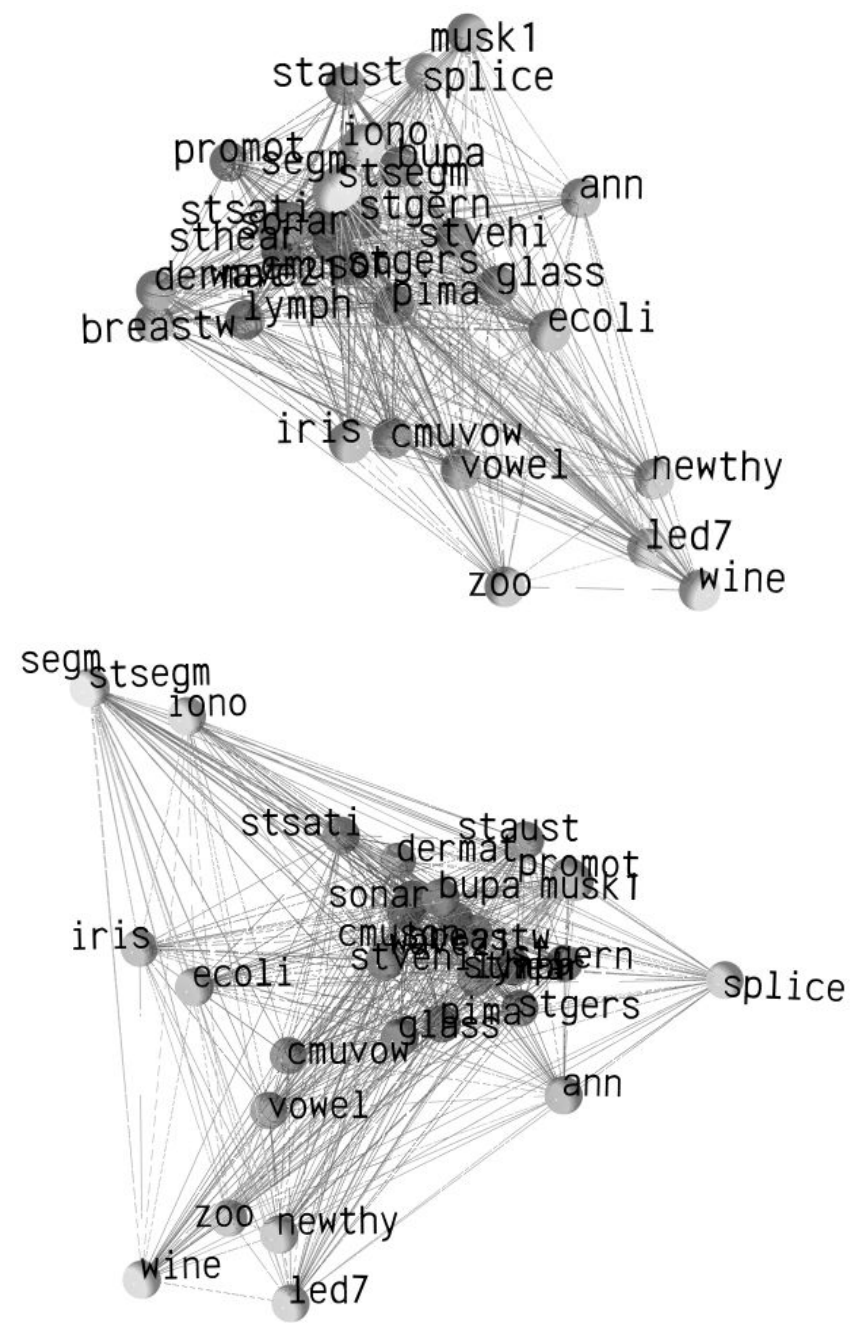

Figure 1: A visualization of task space in three dimensions for thirty tasks from the Irvine Machine Learning Repository. Two views of the model are shown, roughly 90 degrees apart. 


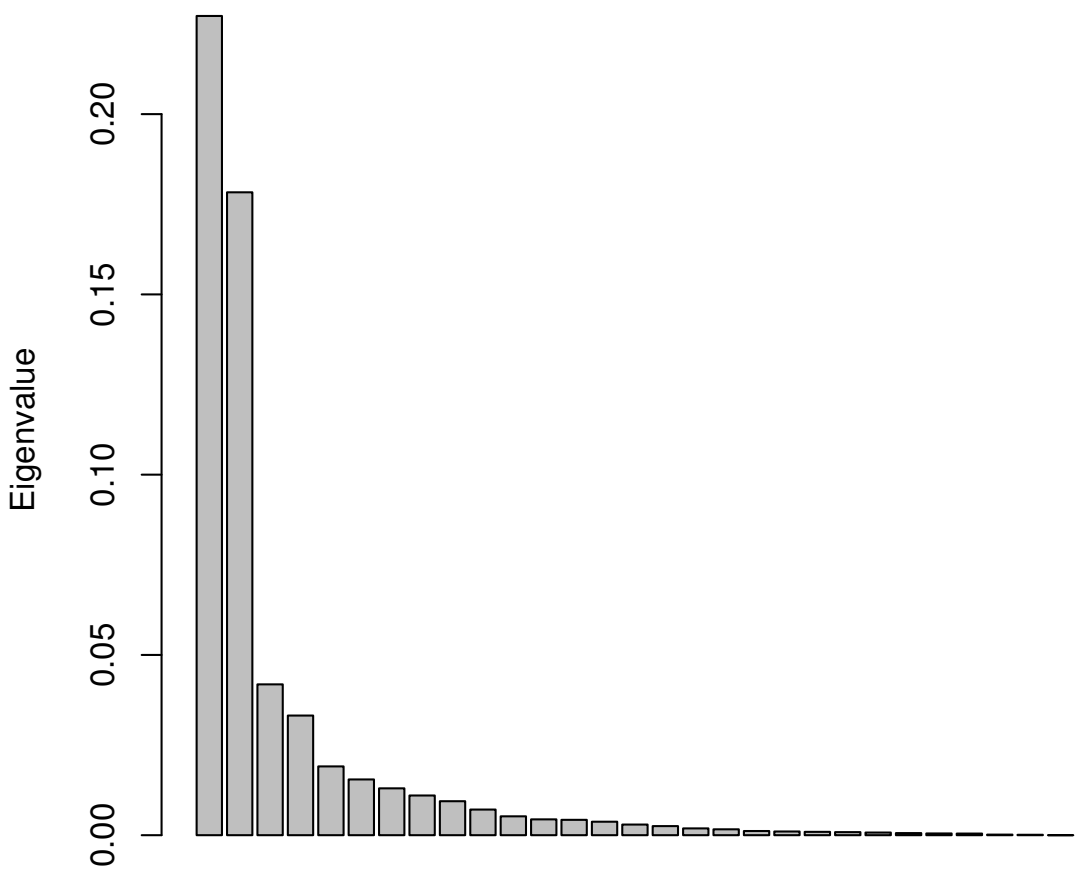

Figure 2: The eigenvalues of the task space's covariance matrix. 


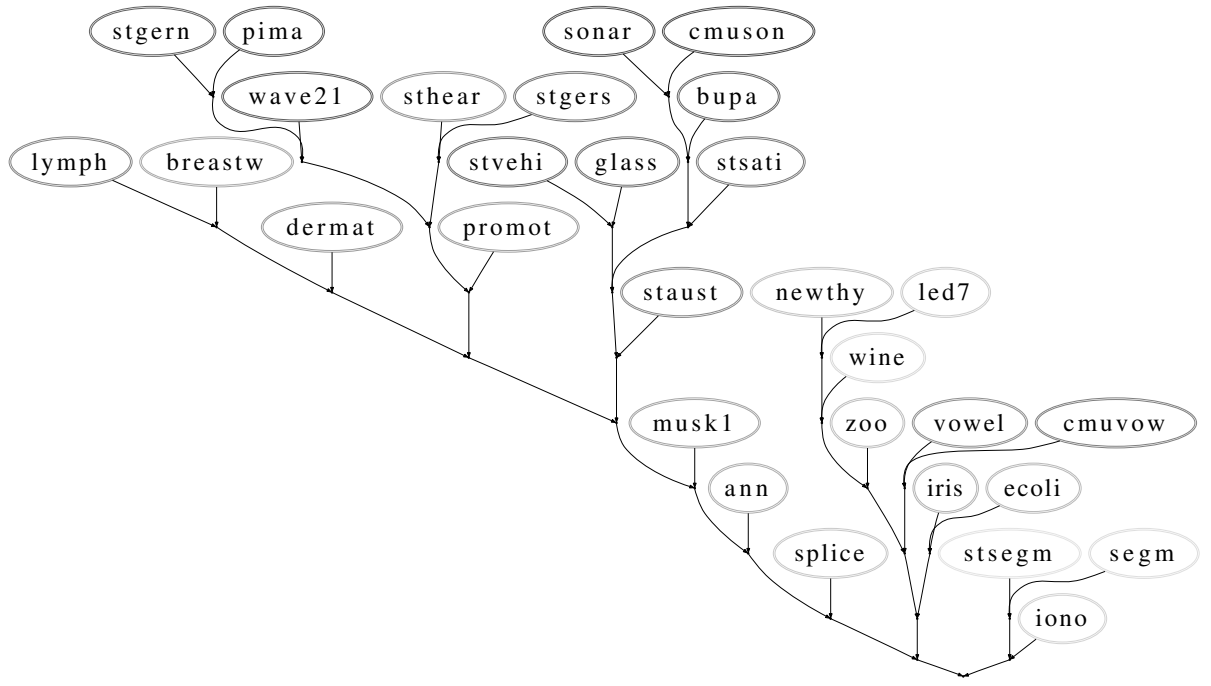

Figure 3: Clustering of learning tasks using Average Link Agglomerative Clustering. 